\title{
ICT Requirements Analysis for Enterprise Networks Supporting Solar Power Plants
}

\author{
Hamideh Afsarmanesh $^{1}$ and Victor Thamburaj ${ }^{2}$ \\ ${ }^{1}$ University of Amsterdam, Informatics Institute, FCN group, \\ Science Park 107, 1098 XG Amsterdam, The Netherlands \\ h. afsarmanesh@uva.nl \\ ${ }^{2}$ iPLON GmbH, The Infranet Company, Karl-Kurz-Str. 36, \\ D- 74523 Schwaebisch Hall, Germany \\ victor. thamburaj@iplon.de
}

\begin{abstract}
Establishing solar power plants are being accepted worldwide as a source of renewable energy. The Total Life Cycle (TLC) of these plants [1] lasts a few decades and includes the three main stages of (i) design and engineering, (ii) construction and commissioning, and the long term (iii) operation and maintenance. A number of different stakeholders are typically involved and contracted that together produce, parameterize, operate, and maintain these plants. Each solar plant is then developed as a complex, one-ofa-kind, massively customized product, which is enhanced by a large number of varied services, either provided as software systems or as business services supported by humans and/or robots. ICT support for the TLC of the solar power plants is challenging. We propose to establish enterprise networks in form of long term alliances, in order to enhance the functionality effectiveness for coworking and co-innovation among the involved stakeholders in this area. This network establishes the common ICT platform and the tools supporting interoperability and secure sharing and exchange of distributed data. The paper provides results from early stages of our goal-oriented requirements engineering [2], mostly focused on domain analysis, requirement elicitation, and stakeholders' verification/agreement. It identifies the high level ICT requirements for this environment, through analyzing its life cycle stages, main entities, stakeholders, and base functionality. This paper concludes with the synthesis of our findings into five classes of ICT requirements.
\end{abstract}

Keywords: Solar power plants, ICT requirement analysis, collaborative networks, Virtual organizations breeding environments, virtual organizations.

\section{Introduction}

Three major factors have been driving the growth in renewable energy generation in the last decade: (i) The main source of energy, fossil fuel, has been depleting fast, but the demand for energy has been growing at tremendous pace, (ii) The burning of fossil fuels has resulted in the emission of huge volumes of Green House Gases (GHG) including $\mathrm{CO}$, which is widely accepted to be the cause of global warming, 
climate change and environmental disasters, and (iii) The political turmoil in countries that are major producers of fossil fuels.

Renewable energy is increasingly considered as a solution to the above problems. Next to the solar panels, a number of other equipment are necessary for construction of solar plants, such as the tables, DC junction Boxes, Inverters, Transformers, electrical switchgear, and grid power connection. But besides the emergy generation related equipment, other fundamental equipment for the solar plants include the monitoring and control equipment which are used by a number of software systems and for performing certain business services. These include systems for control of the operation, management, and maintenance, to ensure smooth production and efficiency of power generation, as well as preserving the legality and security aspects of the solar plants that clearly differ from region to region and country to country.

The primary aim behind the domain analysis phase of our solar plant requirements engineering approach [2] and [3], was to identify the variety of high level ICT requirements for solutions to smoothen collaboration among involved enterprises and stakeholders in the industry, and improving the TLC management of solar plant installations. In this direction, the elicitation process focused on addressing the needed co-innovation, co-creation, and mass customization of service-enhanced products and software systems to meet the stakeholders' needs.

In the remaining of this paper, first in Section 2 we briefly describe the general description of solar power plant environment. This is done along the three phases of its life cycle, as the base for our requirements elicitation purposes. We then address and exemplify in Section 3, the identified stakeholders in this environment, and in Section 4 the need for collaborative development of advanced functionality within this young industry. Section 5 provides a synthesis of the main identified high level ICT requirements for solar power plants, and Section 6 concludes the paper.

\section{Development Phases of Solar Power Plants}

This paper considers the scope of large ground mounted solar power plants (between $1 \mathrm{MWp}$ to $40 \mathrm{MWp}$ ). The Total Life Cycle (TLC) of large power plants consists of three main phases: (a) The phase-1 constitutes design and engineering, typically lasting 3 to 6 months; (b) The phase-2 constitutes construction and commissioning, typically lasting 3 to 6 months; and (c) The phase-3 constitutes Operation and Maintenance of the plant, typically lasting 20 to 25 years.

\section{Development phase-1: Design and Engineering}

This phase is complex and involves various categories of stakeholders. Besides supporting their requirements, the ICT requirement analysis also needs to focus on the needed functionality in this environment, e.g. through a set of business services and software systems. This phase is typically divided into the following three steps:

a) Project Assessment. This step includes a complete analysis of the site and its technical assessment, including: (i) Connection to power GRID (power evacuation 
point), (ii) Technical stability (due diligence) in the area, and (iii) Land field stability (e.g. slopes).

b) Project Design. This step focuses on early engineering, assessment, and selection of technology, including: (i) Pre-engineering (e.g. simulation of distance between tables of solar panels, and arrangement and angels of panels), (ii) Evaluation/selection of solar power technology equipment, and (iii) Selection of inverters (to invert DC from panel to AC for grid).

c) Project Implementation. This step establishes all needed technical connections (e.g. to the Utility company). Furthermore, it finalizes the technology selection and engineering, and the procurement of the needed: equipment, support business services, and software systems, which cover the needed monitoring and control services, as well as the operation and maintenance services for the plant.

\section{Development phase-2: Construction and Commissioning}

During this phase, the solar plant equipment is installed at the site and put into operation. This process is split into two steps of construction and commissioning.

a) Construction. During this step equipment will arrive at the site, and the construction of plant is achieved by different enterprises, ranging from pulling electric cables to installing the monitoring and control equipment. These multi-stakeholder activities need to be carefully coordinated, using project management software.

b) Commissioning. This step deals with testing, adjusting and tuning all control devices and ICT systems, and continues with trouble shooting until the solar plant is successfully started and operational.

\section{Development phase-3: Operation and Maintenance}

The operation and maintenance steps of the solar power plant are intertwined, and focused on efficient generation of electricity, and its transmission to the utility companies, through the evacuation points at the plant. A number of software systems and business services enhance the performance of power plants and assist/guarantee their proper operation [3]. Two main categories of software systems are typically required, namely: (i) the monitoring and control software systems, and (ii) the operational management software systems. The main aim of monitoring a plant is to determine its performance, and weak points, e.g. a malfunctioning device, in which case a flag is raised for maintenance. Furthermore, several software systems at the plant need to interact and exchange information, to coordinate their inter-related functions.

\section{$3 \quad$ Stakeholders in Solar Energy Industry}

Various stakeholders are identified in the solar plant environments, who are either impacted and/or have impact on this area [4]. Many of these stakeholders need to join effort and co-work, while some others have only interest in receiving information about certain features of the plants, e.g. configuration, performance, etc. The categories of identified stakeholders include: Project developing firms, EPC 
(Engineering, Procurement, and Construction) contractors, Lending organizations, Government agencies, Insurance companies, Owner investing firms, Utility companies, equipment manufacturers, Software system developers, Business service providing companies, etc. These stakeholders are typically involved in more than one solar plant, and the plants are often located in different geographical regions. At the same time, at each plant, equipment and devices, the monitoring and control systems, business services, etc. are provided by different specialized enterprises, which frequently need to work jointly. For example, several solar plants in India are now being designed and engineered in Germany [5]. As such clearly the mentioned processes in Section 2 become quite challenging and may involve collaboration among very large number of supporting SME organizations, from different locations, what calls for establishing collaborative network, as addressed in Section 7.

\section{Need for Collaborative Development of Advanced Functionality}

Solar energy is a young industry, in which supporting enterprises, e.g. developers of operation and maintenance systems, must continuously innovate and propose new services in response to the identified needs and/or raised problems by different stakeholders. Therefore, as a part of our requirements analysis [6], besides the general ICT requirements mentioned above, we identify and analyze the need for provision of advanced services and functionality in this industry. These are mostly related to cocreation, co-innovation, and mass-customization functionality, while involving customers. This section addresses a real case example, to demonstrate the need for innovation through co-development within collaborative networks. The case is about a solar plant built by German companies in the desert in Gujarat, India [5]. The customer at this plant raised the problem that the solar panels are frequently covered with dust, hence hindering their effective electric power generation capabilities. Among the main challenges were: finding necessary clean water in the desert, and training the local staff at the site to safely clean the panels (which generates $1000 \mathrm{~V}$ DC) while avoiding electric shock. To find an innovative solution needed a group of companies partly in Germany and partly in India to collaborate in designing a novel semi-automatic cleaning system. The required expertise for this innovation included: (i) Mechanical cleaning tools, with brushes and clamps, (ii) Sensitive chemicals to not damage the panels or environment, (iii) Collecting used water from different sources, (iv) Recycling used water, (v) Control systems for measurement of dust before and after cleaning, (vi) Safety checking device for humans cleaning panels, and (vii) Developing multimedia training tools (with audio, video, and text). The designed approach uses waste-water from close-by homes and industries to be recycled for cleaning dusty panels, and design of a new specialized equipment.

Once this semi-automatic cleaning system is developed and tested at different sites , with varied requirements, it will be provided in the market with mass customization features to fit the requirements at different plants. This means that in future customers can buy this cleaning equipment, and remotely download the customization software 
needed to parameterize/tailor/configure the cleaning system to fit their specific needs and preferences, and become a part of their operation and maintenance services.

\section{$5 \quad$ Analysis and Synthesis of Main Identified Requirements}

The solar power plant domain embeds many complexities that can benefit from ICTbased developments. Our main identified requirements are analyzed and classified below into four requirement classes, addressed below. These synthesized requirements are verified/validated by experts in the field of solar power plants [5].

\section{$\underline{\text { Requirement Class } 1 \text { - Networking among Involved Stakeholder Organizations }}$}

Considering the variety of involved stakeholders, and their needed interactions and information sharing/exchange, establishment of formal long term strategic alliance networks is required to serve as the base infrastructure for their collaboration. Some forms of informal networking already exists in this industry, but it is necessary to establish a so-called VBEs (Virtual organizations Breeding Environments) [7] in support of collaborative activities in this industry. The VBE alliance brings together and supports collaboration among otherwise independent and mostly small organizations, e.g. those providing the needed equipment, business services, and software systems, etc. with decision makers, and even customers when they are included within innovation loops. At present, these stakeholders need to collaborate on different tasks, from simple service delivery to co-construction, and even to increasing the potential of turning a customer need into innovation. Within such a VBE, a number of goal-oriented Virtual Organizations (VOs) can be dynamically established, each aimed to fulfill specific joint task by a group of enterprises, e.g. a joint construction or commissioning task, or certain co-creation/co-innovation activity. Fig. 1 represents different involved stakeholders and, their relation with EPC, plus our proposed high level networking interrelations through the VBE and VOs.

Through establishing VBEs [7], its member stakeholders can together: (i) capture bigger market, and more opportunities, (ii) reduce individual costs, by each focusing only on its core competencies, and (iii) increase individual abilities to take risks, through distribution and sharing of their profits and losses.

Research on VBEs has so far addressed a number of ICT supporting tools. Among them, the base functionality that are identified as required for the solar energy domain includes the following: (i) Catalog of member profiles [8] identifying who is who in the VBE \& what are their competencies, (ii) Performance-based trust establishment [9] among the VBE stakeholders, (iii) A glossary and/or ontology of terms/concepts [10] to define the common terminology related to the energy area, and (iv) Common set of basic tools for goal-oriented collaboration among VBE members including: the Most-fit partner selection tool, for establishing the so called opportunity-based VOs (Virtual Organizations) [11], and the Negotiation tool, to record agreements on responsibilities, liabilities, and distribution of the profits and losses. For the case of service-enhanced complex products, every one of the above mentioned functionality are needed to be carefully researched and developed. 


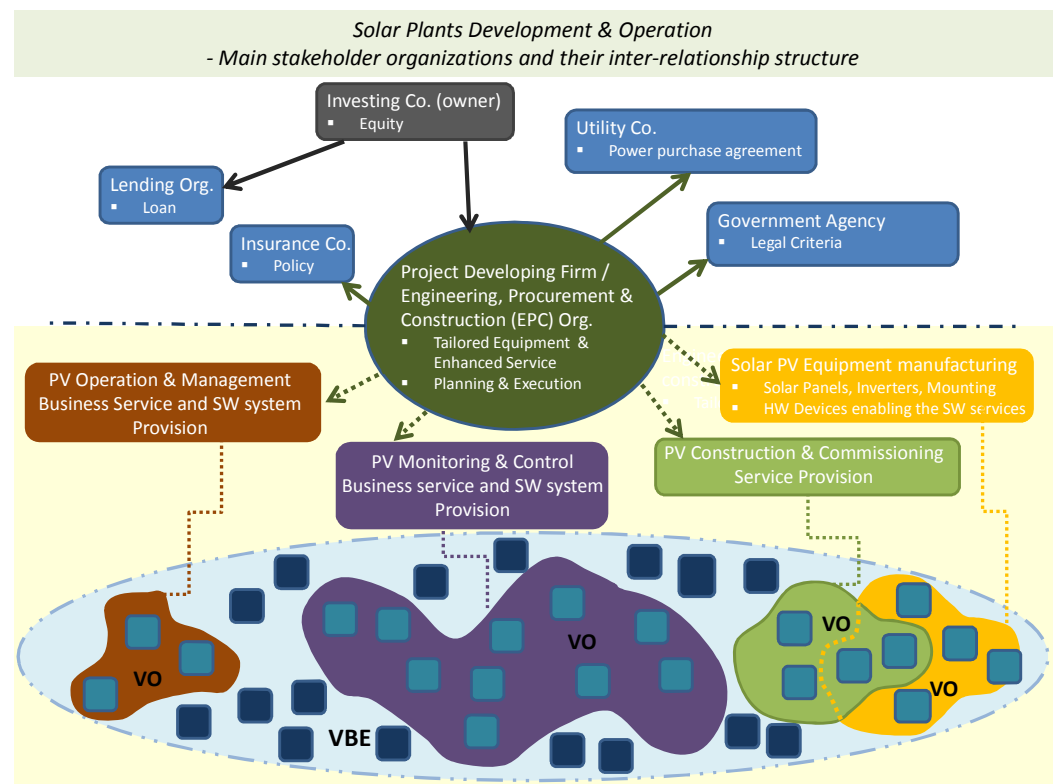

Fig. 1. Main Stakeholders \& Enterprise Networks

\section{Requirement Class 2 - Infrastructure for Co-working \& Co-development}

Currently ICT is used minimally for collaboration in energy area. The needed collaboration is rendered through simple means of communication, such as telephone, fax, and email. And although greatly needed, there is no common ICT framework where the needed sharing and collaboration can be supported, i.e. a framework where joint services can be developed, provided, channeled, and offered as a complete solution to customer. For this purpose, a supportive ICT infrastructure is required to be developed and tailored to the identified needs. The infrastructure shall preferably be based on de-facto standards, providing a base sharing platform and tools through which the information/knowledge, as well as software systems, can be easily provided and effectively retrieved and accessed by any authorized user at any geographic location. Our analysis of identified requirements has revealed that cloud computing infrastructure can serve as the base communication and sharing infrastructure [12] for this domain, the service oriented architecture [13] can serve as the foundation for ICT developments, while web services can support the uniform creation, aggregation, and delivery of data/knowledge through the web to all the stakeholders.

Following are the main requirements for this needed sharing infrastructure and its support tools: (i) Communication infrastructure shall provide: cost effective solution, preserving security/privacy, access by authorized users, ease of use by different stakeholders, timely transfer of sensored data (in pseudo real time); (ii) Interoperability infrastructure shall support: sharing/integration of information/knowledge, discovery and composition of software components in support of developing integrated business services; (iii) Multi-media based infrastructure shall support multi-user co-design. 
Requirement Class 3 - Shared Information/Knowledge and Software System Assets (within the VBEs)

Once operational, the energy-related VBEs gradually build up a large set of information/knowledge assets, during the operation/maintenance phase of their life cycles. we have identified ten main such entities about which their information / knowledge need to be stored and shared with the VBE, that constitute a virtual Bag Of Information/Knowledge Assets (BOIKA). These entities include: equipment, support services, stakeholders, orders history, brochures, products, historic sensored data, generated knowledge - represented as processes (e.g. best practice, lessons learned, etc.), product-based VBE network, and the VO networks. Besides the BOIKA, this domain generated innovative software systems that can be shared and integrated into more value added solutions. But for sharing purposes, innovate approaches need to be developed to formalize the syntax, semantics, and behavior of the software modules - e.g. applying formalisms such as the UML for semantics[14], WSDL for syntax [15], and constraint automata for behavior [16], in order to make them machine readable, needed for their discovery and potentially their semiautomated composition into integrated systems. Such system are in principle applied to: (i) enhancing the abilities and performance of different equipment in the plant (e.g. advanced monitoring systems enhancing the camera equipment), and (ii) managing the operation of the plants (e.g. event handling systems).

Further to the requirement for an information system to organize and storage these information/knowledge assets in the VBE, in order for them to be shared within the community, other mechanisms and ICT tools with user-friendly interfaces and editors are required to support: (i) Search, retrieve, and update of assets; (ii) Protecting the privacy of community assets, while providing authorized shared access; and (iii) Developing incentives for contribution to the sharable bag of community assets and supporting their providers.

\section{Requirement Class 4 - Semi-automated Learning-Based Decision Support Tools Assisting Stakeholders}

Continuous collecting, monitoring, and analysis of the vast amount of historic information and knowledge, and sensored data is required in the plants. It is necessary to build tools to assist users with their complex decision making tasks in this environment, and aiming to partially automate decision making. Stakeholders in this domain express uncertainty and need to use intuition and/or partial information when making some decisions in this domain. Simultaneously, large amount of historic data exists at plants with many relevant parameters that if processed, can assist with making accurate decisions. Development of the following two machine-learning based systems are identified as required to assist stakeholders: (i) Semi-automated assisting tool for power plant product configuration - learning based techniques to gradually learn from analysis of data related to establishment of past power plants; (ii) Semi-automated assisting tool for power plant performance enhancement \& fault prognosis - learning from data about past repair reports and discovering their relationships to past collected sensored/ monitored performance data at the plant. For instance to identify potentially malfunctioning components in the plan or 
forecasting needed repair, before the actual break down of such components; thus using the techniques for prognosis of faults at the power plant.

\section{Concluding Remarks}

This paper presents the ICT requirements analysis results performed within the solar power plant area. It describes the main stages of solar power plants life cycle and identifies the main roles of various stakeholders in this domain. It also discusses the need to support challenging functionality in this domain, e.g. co-innovation among geographically dispersed organizations. The main identified ICT requirements are then analyzed and synthesized, which is validated by experts in the field.

Acknowledgements. The work on this paper is partially supported by the FP7 project GLONET, funded by the European Commission. We also thank the members of the GLONET consortium for the fruitful discussion on service enhanced products, such as solar power plants.

\section{References}

1. Methodology Guidelines on Life Cycle Assessment of Photovoltaic Electricity, http://www.clca.columbia.edu/IEA_Task12_LCA_Guidelines_ 12_1_11_Latest.pdf

2. Ross, D., Schoman, K.: Structured Analysis for Requirements Definition. IEEE Transactions on Software Engineering 3(1), 6-15 (1977)

3. Addressing Solar Photovoltaic Operations and Maintanance Challenges (2010), http: / /www. smartgridnews. com/artman/uploads/1/ 1021496AddressingPVOaMChallenges7-2010_1_.pdf

4. Nuseibeh, B., Easterbrook, S.: Requirements Engineering: A Roadmap. In: Proc. Conference on the Future of Software Engineering, Ireland, pp. 279-289 (2000)

5. Afsarmanesh, H., Thamburaj, V.: Detailed Requirements for GloNet use case and Domain Glossary. EU Project GloNet (285273) - Deliverable 1.1, Global enterprise network focucing on customer-centric collaboration (2012)

6. Zave, P.: Classification of Research Efforts in Requirements Engineering. ACM Computing Surveys 29(4), 315-321 (1997)

7. Afsarmanesh, H., Camarinha-Matos, L.M., Msanjila, S.S.: Models, Methodologies, and Tools Supporting Establishment and Management of Second-Generation VBEs. IEEE Transactions on Systems, Man, and Cybernetics, Part C: Applications and Reviews 41(5), 692-710 (2011)

8. Afsarmanesh, H., Ermilova, E.: Competency modeling targeted on boosting configuration of Virtual Organizations. International Journal of Production Planning \& Control, special issue on Engagement in Collaborative Networks 21(2), 103-118 (2010)

9. Msanjila, S.S., Afsarmanesh, H.: FETR: A Framework to Establish Trust Relationships among Organizations in VBEs. Journal of Intelligent Manufacturing 21(3), 251-265 (2010) 
10. Afsarmanesh, H., Ermilova, E.: The Management of Ontologies in the VO Breeding Environments Domain. International Journal of Services and Operations Management IJSOM, Special Issue on Modelling and Management of Knowledge in Collaborative Networks 6(3), 257-292 (2010)

11. Camarinha-Matos, L.M., Afsarmanesh, H.: Collaborative networks: A new scientific discipline. Intelligent Manufacturing 16, 439-452 (2005)

12. Eight ways that cloud computing will change business (2009), http: / / www. zdnet.com/blog/hinchcliffe/ eight-ways-that-cloud-computing-will-change-business / 488

13. Arbab, F.: Will the Real Service Oriented Computing Please Stand Up? In: Barbosa, L.S., Lunpe, M. (eds.) FACS 2010. LNCS, vol. 6921, pp. 277-285. Springer, Heidelberg (2012)

14. Unified Modeling Language (UML), http: / / www . uml . org /

15. Web Service Description Language (WSDL), http: / /www . w3 . org/TR/wsdl

16. Constraint automata, http://reo.project.cwi.nl/ cgi-bin/trac.cgi/reo/wiki/ConstraintAutomata 